\title{
Growth enhancement and protective potential of feed-based outer membrane proteins against vibriosis in Macrobrachium rosenbergii
}

\begin{abstract}
ABSTRCT
The use of antibiotics to curtail vibriosis, which is a major infectious disease, plaguing shrimp and prawn is rather becoming less effective and the need for a better alternative is expedient. The outer membrane proteins (OMPs) of V. alginolyticus were extracted, mixed with powdered commercial feed and fed to the prawns to evaluate its effect on growth performance and protective potential. Sixty prawns were divided into groups A, B and C of 10 prawns each, with two replicates in six $(150 \mathrm{~L})$ glass aquaria. Groups A, B and C were fed with OMPs mixed diet, with OMPs-Freund's incomplete adjuvant mixed diet and OMPs or adjuvant free diet (control diet) respectively. All the prawns were weighed weekly, and haemolymph was collected to determine the total haemocyte count (THC) and phenoloxidase (PO) activity. At the end of the feeding trial, prawns were intramuscularly challenged with $50 \mu \mathrm{L}$ of $107 \mathrm{CFU} \mathrm{V}$. alginolyticus. The treated groups were significantly higher in growth performance and THC than the control group, but no significant difference between the groups in terms of PO activity and mortality rate. The study, however, submitted that oral administration of OMPs with or without adjuvant is a good growth promoter and has the potential for protection against vibriosis in giant freshwater prawn (Macrobrachium rosenbergii).
\end{abstract}

.Keyword: Vibriosis; Macrobrachium rosenbergii; OMPs; Growth; Protection. 